\title{
Measurement of the tau lepton lifetime
}

\section{OPAL Collaboration}

P.D. Acton ${ }^{\text {a }}$, G. Alexander ${ }^{\text {b }}$, J. Allison ${ }^{\text {c }}$, P.P. Allport ${ }^{\text {d }}$, K.J. Anderson ${ }^{\mathrm{e}}$, S. Arcelli ${ }^{\mathrm{f}}$, P. Ashton ${ }^{\mathrm{c}}$, A. Astbury 1, D. Axen ${ }^{2}$, G. Azuelos ${ }^{\text {g, }}$, G.A. Bahan ${ }^{\text {c }}$, J.T.M. Baines ${ }^{\text {c }}$, A.H. Ball ${ }^{\text {h }}$, J. Banks ${ }^{c}$, G.J. Barker ${ }^{\text {i }}$, R.J. Barlow ${ }^{\text {c }}$, J.R. Batley ${ }^{d}$, G. Beaudoin ${ }^{\mathrm{g}}$, A. Beck ${ }^{\mathrm{b}}$, J. Becker ${ }^{\mathrm{j}}$, T. Behnke ${ }^{\mathrm{k}}$, K.W. Bell ${ }^{\ell}$, G. Bella ${ }^{b}$, P. Berlich ${ }^{j}$, S. Bethke ${ }^{m}$, O. Biebel $^{n}$, U. Binder ${ }^{j}$, I.J. Bloodworth ${ }^{\circ}$, P. Bock ${ }^{\mathrm{m}}$, B. Boden $^{\mathrm{n}}$, H.M. Bosch ${ }^{\mathrm{m}}$, S. Bougerolle $^{2}$, B.B. Brabson ${ }^{\mathrm{p}}$, H. Breuker $^{\mathrm{q}}$, R.M. Brown ${ }^{\ell}$, R. Brun ${ }^{\mathrm{q}}$, A. Buijs ${ }^{\mathrm{q}}$, H.J. Burckhart ${ }^{\mathrm{q}}$, P. Capiluppi ${ }^{\mathrm{f}}$, R.K. Carnegie ${ }^{\mathrm{r}}$, A.A. Carter ${ }^{i}$, J.R. Carter ${ }^{\text {d }}$, C.Y. Chang ${ }^{\text {h }}$, D.G. Charlton ${ }^{\text {q }}$, P.E.L. Clarke ${ }^{\mathrm{a}}$, I. Cohen ${ }^{\mathrm{b}}$, W.J. Collins ${ }^{d}$, J.E. Conboy ${ }^{s}$, M. Cooper ${ }^{t}$, M. Couch ${ }^{\circ}$, M. Coupland ${ }^{\mathrm{u}}$, M. Cuffiani ${ }^{\mathrm{f}}$, S. Dado $^{t}$, G.M. Dallavalle ${ }^{f}$, S. De Jong ${ }^{q}$, P. Debu ${ }^{v}$, L.A. del Pozo ${ }^{d}$, M.M. Deninno ${ }^{f}$, A. Dieckmann ${ }^{m}$, M. Dittmar ${ }^{w}$, M.S. Dixit ${ }^{x}$, E. Duchovni ${ }^{y}$, G. Duckeck ${ }^{\mathrm{m}}$, I.P. Duerdoth ${ }^{\mathrm{c}}$, D.J.P. Dumas ${ }^{\mathrm{r}}$, G. Eckerlin ${ }^{\mathrm{m}}$, P.A. Elcombe ${ }^{\mathrm{d}}$, P.G. Estabrooks ${ }^{\mathrm{r}}$, E. Etzion ${ }^{\mathrm{b}}$, F. Fabbri ${ }^{\mathrm{f}}$, M. Fincke-Keeler ${ }^{1}$, H.M. Fischer ${ }^{\text {n }}$, D.G. Fong ${ }^{\text {h }}$, C. Fukunaga ${ }^{z}$, A. Gaidot ${ }^{\text {v }}$, O. Ganel ${ }^{y}$, J.W. Gary ${ }^{\text {w }}$, J. Gascon ${ }^{g}$, R.F. McGowan ${ }^{\text {, }}$, N.I. Geddes ${ }^{\ell}$, C. Geich-Gimbel ${ }^{\text {n }}$, S.W. Gensler ${ }^{\mathrm{e}}$, F.X. Gentit ${ }^{\mathrm{v}}$, G. Giacomellif ${ }^{\text {, V. Vibson }}{ }^{\mathrm{d}}$, W.R. Gibson ${ }^{\mathrm{i}}$, J.D. Gillies ${ }^{\ell}$, J. Goldberg ${ }^{\mathrm{t}}$, M.J. Goodrick ${ }^{\mathrm{d}}$, W. Gorn ${ }^{\text {w }}$, C. Grandi ${ }^{\text {f }}$, F.C. Grant ${ }^{\text {d }}$, J. Hagemann ${ }^{\text {k }}$, G.G. Hanson ${ }^{\text {p }}$, M. Hansroul ${ }^{\text {, }}$, C.K. Hargrove ${ }^{x}$, P.F. Harrison ${ }^{i}$, J. Hart ${ }^{d}$, P.M. Hattersley ${ }^{\circ}$, M. Hauschild ${ }^{q}$, C.M. Hawkes ${ }^{q}$, E. Heflin ${ }^{w}$, R.J. Hemingway ${ }^{r}$, R.D. Heuer ${ }^{\text {q }}$, J.C. Hill ${ }^{d}$, S.J. Hillier ${ }^{\circ}$, D.A. Hinshaw ${ }^{g}$, C. Ho $^{\text {w }}$, J.D. Hobbs ${ }^{q}$, P.R. Hobson ${ }^{\text {a }}$, D. Hochman ${ }^{y}$, B. Holl ${ }^{\text {q }}$, R.J. Homer ${ }^{\circ}$, A.K. Honma ${ }^{4}$, S.R. Hou ${ }^{\text {, }}$, C.P. Howarth ${ }^{\mathrm{s}}$, R.E. Hughes-Jones ${ }^{c}$, R. Humbert ${ }^{\mathrm{j}}$, P. Igo-Kemenes ${ }^{\mathrm{m}}$, H. Ihssen ${ }^{\mathrm{m}}$, D.C. Imrie ${ }^{\mathrm{a}}$, A.C. Janissen ${ }^{\mathrm{r}}$, A. Jawahery ${ }^{\mathrm{h}}$, P.W. Jeffreys ${ }^{\ell}$, H. Jeremie ${ }^{\mathrm{g}}$, M. Jimack ${ }^{\mathrm{f}}$, M. Jobes ${ }^{\mathrm{o}}$, R.W.L. Jones ${ }^{\text {i }}$, P. Jovanovic ${ }^{\circ}$, D. Karlen ${ }^{\text {r }}$, K. Kawagoe ${ }^{z}$, T. Kawamoto ${ }^{\text {, }}$, R.K. Keeler ${ }^{1}$, R.G. Kellogg ${ }^{\text {n }}$, B.W. Kennedy ${ }^{\text {s }}$, C. Kleinwort ${ }^{\text {q }}$, D.E. Klem ${ }^{\text {aa }}$, T. Kobayashi ${ }^{z}$, T.P. Kokott ${ }^{\text {n, }}$ S. Komamiya ${ }^{z}$, L. Köpke ${ }^{\text {q }}$, J.F. Kral ${ }^{\text {q }}$, R. Kowalewski ${ }^{r}$, H. Kreutzmann ${ }^{\text {n }}$, J. von Krogh ${ }^{\text {m }}$, J. Kroll ${ }^{\text {e }}$, M. Kuwano ${ }^{\text {, }}$, P. Kyberd ${ }^{i}$, G.D. Lafferty ${ }^{\text {c }}$, F. Lamarche ${ }^{\text {g }}$, W.J. Larson w, J.G. Layter ${ }^{\text {w }}$, P. Le Du ${ }^{\text {v }}$, P. Leblanc ${ }^{\text {g }}$, A.M. Lee ${ }^{\text {h }}$, M.H. Lehto ${ }^{\text {s }}$, D. Lellouch ${ }^{\mathrm{y}}$, P. Lennert ${ }^{\mathrm{m}}$, C. Leroy ${ }^{g}$, J. Letts $^{w}$, S. Levegrün ${ }^{n}$, L. Levinson ${ }^{y}$, S.L. Lloyd ${ }^{\mathrm{i}}$, F.K. Loebinger ${ }^{\mathrm{c}}$, J.M. Lorah ${ }^{\mathrm{h}}$,

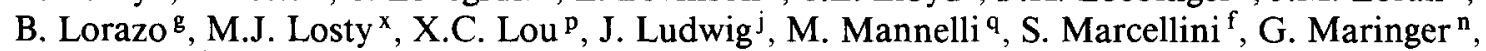
A.J. Martin ${ }^{\text {i }}$, J.P. Marting, T. Mashimo ${ }^{\text {z }}$, P. Mättig ${ }^{\text {n }}$, U. Maur ${ }^{\text {n }}$, J. McKenna ${ }^{1}$, T.J. McMahon ${ }^{\circ}$, J.R. McNutt ${ }^{a}$, F. Meijers ${ }^{q}$, D. Menszner ${ }^{m}$, F.S. Merritt ${ }^{\mathrm{e}}$, H. Mes ${ }^{\mathrm{x}}$, A. Michelini ${ }^{q}$, R.P. Middleton ${ }^{\ell}$, G. Mikenberg ${ }^{y}$, J. Mildenberger $^{r}$, D.J. Miller ${ }^{\mathrm{s}}$, R. Mir $^{p}$, W. Mohr ${ }^{j}$, C. Moisan ${ }^{g}$, A. Montanarif ${ }^{f}$, T. Mori ${ }^{z}$, M.W. Moss ${ }^{c}$, T. Mouthuy ${ }^{p}$, B. Nellen ${ }^{n}$, H.H. Nguyen ${ }^{\mathrm{e}}$, M. Nozaki ${ }^{\mathrm{z}}$, S.W. O'Neale ${ }^{\mathrm{q}, 5}{ }^{5}$, B.P. O’Neill ${ }^{\mathrm{w}}$, F.G. Oakham ${ }^{\mathrm{x}}$, F. Odorici ${ }^{\mathrm{f}}$, M. Ogg ${ }^{r}$, H.O. Ogren ${ }^{\text {p }}$, H. Oh ${ }^{\text {w }}$, C.J. Oram ${ }^{4}$, M.J. Oreglia ${ }^{\mathrm{e}}$, S. Orito ${ }^{\mathrm{z}}$, J.P. Pansart ${ }^{\mathrm{v}}$, B. Panzer-Steindel ${ }^{\mathrm{q}}$, P. Paschievici ${ }^{\mathrm{y}}$, G.N. Patrick ${ }^{\ell}$, S.J. Pawley ${ }^{\mathrm{c}}$, P. Pfister ${ }^{\mathrm{j}}$, J.E. Pilcher $^{\mathrm{e}}$, J.L. Pinfold ${ }^{y}$, D. Pitman ${ }^{1}$, D.E. Plane ${ }^{\mathrm{q}}$, P. Poffenberger ${ }^{1}$, B. Poli ${ }^{f}$, A. Pouladdej ${ }^{\mathrm{r}}$, E. Prebys ${ }^{\mathrm{q}}$, T.W. Pritchard ${ }^{\text {i }}$, H. Przysiezniak ${ }^{\text {g }}$, G. Quast ${ }^{k}$, M.W. Redmond ${ }^{\mathrm{e}}$, D.L. Rees ${ }^{\circ}$, K. Riles $^{\mathrm{w}}$, S.A. Robins ${ }^{i}$, D. Robinson ${ }^{\text {q }}$, A. Rollnik ${ }^{\text {n }}$, J.M. Roney ${ }^{\text {e }}$, E. Ros ${ }^{\mathrm{q}}$, S. Rossberg ${ }^{\mathrm{j}}$, A.M. Rossi ${ }^{\mathrm{f}, 6}{ }^{\text {, }}$ M. Rosvick ${ }^{1}$, P. Routenburg ${ }^{r}$, K. Runge ${ }^{j}$, O. Runolfsson ${ }^{\mathrm{q}}$, D.R. Rust ${ }^{\mathrm{p}}$, S. Sanghera ${ }^{\mathrm{r}}$, M. Sasaki ${ }^{\text {}}$, A.D. Schaile ${ }^{j}$, O. Schaile ${ }^{j}$, W. Schappert ${ }^{r}$, P. Scharff-Hansen ${ }^{\text {q }}$, P. Schenk ${ }^{1}$, H. von der Schmitt ${ }^{m}$, S. Schreiber ${ }^{n}$, J. Schwiening ${ }^{n}$, W.G. Scott ${ }^{\ell}$, M. Settles ${ }^{\text {p }}$, B.C. Shen ${ }^{w}$, P. Sherwood ${ }^{\text {s }}$, R. Shypit ${ }^{2}$, A. Simon ${ }^{n}$, P. Singh ${ }^{i}$, G.P. Siroli ${ }^{\text {f }}$ A. Skuja ${ }^{\text {h }}$, A.M. Smith ${ }^{\text {q }}$, 
T.J. Smith ${ }^{\text {q }}$, G.A. Snow ${ }^{\text {h }}$, R. Sobie ${ }^{7}$, R.W. Springer ${ }^{\text {h }}$, M. Sproston ${ }^{\ell}$, K. Stephens ${ }^{\text {c }}$, H.E. Stier ${ }^{\mathrm{j}, 8}$, R. Ströhmer ${ }^{\mathrm{m}}$, D. Strom ${ }^{\mathrm{e}}$, H. Takeda ${ }^{\mathrm{z}}$, T. Takeshita ${ }^{\mathrm{z}}$, P. Taras ${ }^{\mathrm{g}}$, S. Tarem $^{\mathrm{y}}$, P. Teixeira-Dias ${ }^{\mathrm{m}}$, N.J. Thackray $^{\circ}$, G. Transtromer ${ }^{a}$, T. Tsukamoto ${ }^{z}$, M.F. Turner $^{d}$, G. Tysarczyk-Niemeyer ${ }^{\mathrm{m}}$, D. Van den plas ${ }^{\mathrm{g}}$, R. Van Kooten ${ }^{\mathrm{q}}$, G.J. VanDalen ${ }^{\text {w }}$, G. Vasseur ${ }^{\mathrm{v}}$, C.J. Virtue ${ }^{a}$, A. Wagner ${ }^{k}$, C. Wahl ${ }^{j}$, J.P. Walker ${ }^{\circ}$, C.P. Ward ${ }^{d}$, D.R. Ward ${ }^{d}$, P.M. Watkins ${ }^{o}$, A.T. Watson ${ }^{\circ}$, N.K. Watson ${ }^{\mathrm{q}}$, M. Weber ${ }^{\mathrm{m}}$, P. Weber ${ }^{\mathrm{r}}$, S. Weisz ${ }^{\mathrm{q}}$, P.S. Wells ${ }^{\mathrm{q}}$, N. Wermes ${ }^{\mathrm{m}}$, M. Weymann ${ }^{q}$, M.A. Whalley ${ }^{\circ}$, G.W. Wilson ${ }^{v}, J^{\prime A}$. Wilson ${ }^{\circ}$, I. Wingerter ${ }^{q}$, V.-H. Winterer ${ }^{j}$, N.C. Wood ${ }^{c}$, S. Wotton ${ }^{q}$, T.R. Wyatt ${ }^{c}$, R. Yaari ${ }^{y}$, Y. Yang ${ }^{w, 9}$, G. Yekutieli ${ }^{y}$, M. Yurko ${ }^{g}$, I. Zacharov ${ }^{\mathrm{q}}$, W. Zeuner ${ }^{\mathrm{q}}$ and G.T. Zorn ${ }^{\mathrm{h}}$

${ }^{a}$ Brunel University, Uxbridge, Middlesex $U B 83 P H, U K$

b Department of Physics and Astronomy, Tel Aviv University, Tel Aviv 69978, Israel

' Department of Physics, Schuster Laboratory, The University, Manchester M13 9PL, UK

d Cavendish Laboratory, Cambridge CB3 OHE, UK

e Enrico Fermi Institute and Department of Physics, University of Chicago, Chicago, IL 60637, USA

f Dipartimento di Fisica dell' Università di Bologna and INFN, I-40126 Bologna, Italy

${ }^{8}$ Laboratoire de Physique Nucléaire, Université de Montréal, Montreal, Quebec, Canada H3C 3J7

h Department of Physics and Astronomy, University of Maryland, College Park, MD 20742, USA

i Queen Mary and Westfield College, University of London, London E1 4NS, UK

j Fakultät für Physik, Albert Ludwigs Universität, W-7800 Freiburg, FRG

k Universität Hamburg/DESY, II. Institut für Experimental Physik, W-2000 Hamburg 52, FRG

Rutherford Appleton Laboratory, Chilton, Didcot, Oxfordshire OX11 0QX, UK

m Physikalisches Institut, Universität Heidelberg, W-6900 Heidelberg, FRG

n Physikalisches Institut, Universität Bonn, W-5300 Bonn 1, FRG

- School of Physics and Space Research, University of Birmingham, Birmingham B15 2TT, UK

p Department of Physics, Indiana University, Swain Hall West 117, Bloomington, IN 47405, USA

q CERN, European Organisation for Particle Physics, CH-1211 Geneva 23, Switzerland

r Department of Physics, Carleton University, Colonel By Drive, Ottawa, Ontario, Canada K1S 5B6

s University College London, London WCIE 6BT, UK

t Department of Physics, Technion-Israel Institute of Technology, Haifa 32000, Israel

u Birkbeck College, London WCIE $7 H V$, UK

' DPhPE, CEN-Saclay, F-91191 Gif-sur-Yvette, France

" Department of Physics, University of California, Riverside, CA 92521, USA

* Centre for Research in Particle Physics, Carleton University, Ottawa, Ontario, Canada K1S 5B6

y Nuclear Physics Department, Weizmann Institute of Science, Rehovot 76100, Israel

${ }^{2}$ International Center for Elementary Particle Physics and Department of Physics, University of Tokyo, Tokyo 113, Japan and Kobe University, Kobe 657, Japan

aa National Research Council of Canada, Herzberg Institute of Astrophysics, Ottawa, Ontario, Canada K1A OR6

Received 9 October 1991

The tau lepton lifetime has been measured using two independent techniques; an impact parameter analysis of the 1-prong decays and a decay length analysis of the 3-prong decays. Approximately $5000 \mathrm{Z}^{0}$ decays to $\tau^{+} \tau^{-}$ have been selected from the data collected with the OPAL detector at LEP during 1990. The results of the two statistically independent measurements are, respectively, $\tau_{1}=0.293 \pm 0.013$ (stat.) \pm 0.013 (syst.) ps and $\tau_{3}=$ $0.327 \pm 0.017$ (stat.) \pm 0.011 (syst.) ps. After combining the statistical and systematic errors for each analysis in quadrature, the weighted average lifetime is calculated to be $\tau_{\tau}=0.308 \pm 0.013 \mathrm{ps}$. 


\section{Introduction}

Within the standard model of the weak and electromagnetic interactions, the tau lepton lifetime may be calculated, under the assumptions of lepton universality and massless neutrinos, from a knowledge of the muon lifetime, the muon mass, the tau mass, and the branching ratio of the tau decay to $e \nu \bar{\nu}[1]$. Using the Particle Data Group values for these latter quantities, the tau lifetime is predicted to be $(0.283 \pm$ $0.007)$ ps which is approximately two standard deviations lower than the current world average measurement of $(0.303 \pm 0.008)$ ps [2]. A new measurement is reported of the tau lifetime from the OPAL experiment which is more precise than any previous experiment.

Presented in this letter is a determination of the tau lifetime with two independent techniques using the $\tau^{+} \tau^{-}$data sample obtained with the OPAL detector at LEP during 1990. An impact parameter method has been applied to all 1-prong tau decays, and a decay length analysis has been made with the 3-prong tau decays. The following sections describe features of the OPAL detector relevant to this analysis, the $\tau^{+} \tau^{-}$data selection, the beam spot determination, and the two lifetime analysis techniques.

1 Department of Physics, University of Victoria, P.O. Box 3055, Victoria, BC, Canada V8W 3P6.

2 Department of Physics, University of British Columbia, 6224 Agriculture Road, Vancouver, BC, Canada V6T $1 \mathrm{Z1}$.

3 Also at TRIUMF, Vancouver, Canada V6T 2 A3.

4 Department of Physics, University of Victoria, P.O. Box 1700 , Victoria, BC, Canada V8W 2Y2, and TRIUMF, Vancouver, Canada V6T $2 \mathrm{~A} 3$.

5 On leave from Birmingham University, Birmingham B15 2TT, UK.

6 Present address: Dipartimento di Fisica, Università della Calabria, and INFN, I-87036 Rende, Italy.

7 Department of Physics, University of British Columbia, 6224 Agriculture Road, Vancouver, BC, Canada V6T 2A6, and IPP, High Energy Physics Department, McGill University, 3600 University Street, Montreal, Quebec, Canada H3A 2 T8.

8 Deceased 25th March 1991.

9 On leave from Research Institute for Computer Peripherals, Hangzhou, China.

\section{The OPAL detector}

The OPAL detector is described in detail elsewhere [3]. The coordinate system is defined so that the $z$ axis follows the electron beam direction, and the $x$ axis is in the plane of the LEP ring. The radial coordinate, $r$, is in the $x-y$ plane. The polar and azimuthal angles, $\theta$ and $\phi$, are defined with respect to the $z$ and $x$ axes, respectively.

The measurements of the trajectories and momenta of charged particles are performed with a precision vertex chamber, a jet chamber and $z$-chambers. The cylindrical vertex drift chamber is located between radii 9 and $24 \mathrm{~cm}$. It surrounds an $8 \mathrm{~cm}$ radius, 1.4 $\mathrm{mm}$ thick carbon fibre beam pipe and is subdivided into 36 azimuthal sectors with 12 axial and 6 stereo anode wires each. In the $r-\phi$ plane the average space resolution is $50 \mu \mathrm{m}$ for the first hit and $90 \mu \mathrm{m}$ for subsequent hits on a given anode wire. The jet chamber is a large volume drift chamber, $4 \mathrm{~m}$ long by $3.7 \mathrm{~m}$ in diameter, which is divided into 24 azimuthal sectors. Each sector contains a sense wire plane having 159 axial anode wires. Each of the wires provides threedimensional coordinate measurements, via drift-time measurement in the $r-\phi$ plane and charge-division measurement in the $z$ direction. The average space point resolution in the $r-\phi$ plane is $130 \mu \mathrm{m}$. The jet chamber also provides measurement of $\mathrm{d} E / \mathrm{d} x$ with a resolution of (3-4)\% which allows identification of particles with momenta up to $20 \mathrm{GeV} / c$. In the barrel region, $|\cos \theta|<0.72$, the jet chamber is surrounded by a set of $z$-chambers which gives an accurate $z$ coordinate measurement. The three drift chamber detectors are located inside a 4 bar pressure vessel, which is placed inside a solenoid coil that provides a magnetic field of $0.435 \mathrm{~T}$. The pressure vessel and the coil have an effective thickness of about $2 X_{0} / \sin \theta$ in the region of $|\cos \theta|<0.7$, where $X_{0}$ is one radiation length. The coil is in turn surrounded by a barrel time-of-flight counter array consisting of 160 scintillator bars with phototube readout at both ends. A lead glass electromagnetic calorimeter corresponding to 24.6 radiation lengths and about two hadronic interaction lengths, together with a presampler, measures the positions and energies of showering particles. The magnet return yoke serves as a hadron calorimeter and is instrumented with 9 layers of streamer tubes. The entire detector is surrounded by four layers of drift cham- 
bers for the detection of muons emerging from the hadron calorimeter. End cap detectors provide similar calorimetry and muon detection over nearly the full remaining solid angle.

The tracks are reconstructed using a method that explicitly incorporates the effect of multiple Coulomb scattering, in the detector gaseous volumes, in the discrete material between chambers and in the beam pipe [4]. The momentum resolution of the tracking detectors for $p \approx 45 \mathrm{GeV} / c$ was $\Delta p / p \approx 9 \%$ and the electromagnetic energy resolution was typically $\Delta E / E \approx 3 \%$ for $E \approx 45 \mathrm{GeV}$. The impact parameter resolution in the $r-\phi$ plane, measured using $\mathrm{Z}^{0} \rightarrow \mu^{+} \mu^{-}$and $\mathrm{Z}^{0} \rightarrow \mathrm{e}^{+} \mathrm{e}^{-}$decays, was $40 \mu \mathrm{m}$ for $45 \mathrm{GeV} / c$ tracks; this resolution degrades to $\approx 65 \mu \mathrm{m}$ at $10 \mathrm{GeV} / c$ transverse momentum and to almost 300 $\mu \mathrm{m}$ at $1 \mathrm{GeV} / c$ due to multiple scattering effects.

\section{The $\boldsymbol{\tau}^{+} \tau^{-}$data selection and Monte Carlo simulation}

From the data taken in $1990,5130 \mathrm{e}^{+} \mathrm{e}^{-} \rightarrow \tau^{+} \tau^{-}$ events have been selected representing an integrated luminosity of $6.3 \mathrm{pb}^{-1}$. The selection procedure uses information from the central tracking detectors and electromagnetic calorimetry to identify events with two back-to-back collimated, low multiplicity jets. Time-of-flight measurements have been used to reject cosmic ray events. Events with a large visible energy and two identified muons have been rejected as $\mathrm{e}^{+} \mathrm{e}^{-} \rightarrow \mu^{+} \mu^{-}$. Multihadronic $Z^{0}$ decays have been rejected by imposing requirements on the numbers of charged tracks and electromagnetic clusters. Total energy cuts have allowed a rejection of $\mathrm{e}^{+} \mathrm{e}^{-} \rightarrow \mathrm{e}^{+} \mathrm{e}^{-}$ events and two-photon processes. Full details can be found elsewhere [5]. The angular region of acceptance covers the range $\left|\cos \theta_{\text {thrust }}\right|<0.9$. Relevant to this analysis are those track and cluster definitions used to define the event sample, given in table 1 . They have been chosen to eliminate calorimeter noise hits and to ensure that only well measured charged tracks are used.

For the Monte Carlo studies used in the following analysis, multihadronic events have been generated using both the JETSET [6] and the HERWIG programs [7]. The program BABAMC [8] has been used to generate $\mathrm{e}^{+} \mathrm{e}^{-} \rightarrow \mathrm{e}^{+} \mathrm{e}^{-}$events and KORALZ [9]
Table 1

Track and cluster definitions used in $\tau^{+} \tau^{-}$event selection. The symbols $d_{0}$ and $z_{0}$ are the distances of closest approach that the track makes to the origin, \# hits is the number of measurements along a track. $p_{\mathrm{t}}$ is the track momentum in the plane orthogonal to the beam, and $r_{\min }$ is the radius of the first hit on the track. The symbol $E$ is the measured cluster energy, \# blocks is the number of blocks in the cluster, and $F_{\max }$ is the fraction of the energy in the most energetic block.

\begin{tabular}{ll}
\hline tracks & $\left|d_{0}\right|<1.0 \mathrm{~cm}$ \\
& $\left|z_{0}\right|<50.0 \mathrm{~cm}$ \\
& $\#$ hits $\geqslant 30$ \\
& $p_{1}>0.1 \mathrm{GeV} / c$ \\
& $r_{\min }<75.0 \mathrm{~cm}$ \\
& $E>0.1 \mathrm{GeV}$ \\
& $E>0.2 \mathrm{GeV}$ \\
clusters - barrel & $\#$ blocks $\geqslant 2$ \\
clusters - endcap & $F_{\max }<0.99$ \\
\hline
\end{tabular}

Table 2

Summary of data statistics and background estimates in the 1-prong and 3-prong decay topologies. The background estimation includes both statistical and systematic uncertainties.

\begin{tabular}{lll}
\hline & 1-prong & 3-prong \\
\hline data & 8296 & 1475 \\
two-photon & $62 \pm 10$ & 0 \\
muons & $101 \pm 33$ & 0 \\
Bhabhas & $31 \pm 82$ & 0 \\
hadrons & $5 \pm 51$ & $18 \pm 34$ \\
total bkg & $199 \pm 103$ & $18 \pm 34$ \\
total bkg \% & $2.4 \pm 1.2$ & $1.2 \pm 2.3$ \\
\hline
\end{tabular}

for $\mathrm{e}^{+} \mathrm{e}^{-} \rightarrow \mu^{+} \mu^{-}$and $\mathrm{e}^{+} \mathrm{e}^{-} \rightarrow \tau^{+} \tau^{-}$events. Backgrounds from two-photon processes have been studied using Monte Carlo generated events [10]. The $\tau^{+} \tau^{-}$ acceptance and the level of background from competing reactions have been determined by passing Monte Carlo simulated events through the OPAL reconstruction code and selection algorithms. The combined geometrical acceptance and selection efficiency is estimated to be $75.8 \%$ for the $\tau$ lifetime analysis. Table 2 gives the breakdown of the data and the backgrounds into their contributions to the 1-prong and 3-prong decays.

The OPAL detector response has been simulated using a program that treats in detail the detector geometry and material as well as effects of detector reso- 
lutions and efficiencies [11]. For the $\tau^{+} \tau^{-}$final state, events have been generated with the KORALZ program at a centre of mass energy of $91.160 \mathrm{GeV}$. The program includes multiple QED hard bremsstrahlung and exponentiation of soft photons from the $\mathrm{e}^{+} \mathrm{e}^{-}$ initial state and also single photon bremsstrahlung from the final state fermions. One large sample of 50000 events has been produced with lifetime equal to the current world average of 0.303 ps. In addition, 9 smaller samples of 5000 events each have been produced with lifetimes ranging from 0.0 to $0.606 \mathrm{ps}$.

\section{The beam spot determination}

The determination of the coordinates of the average beam position proceeds as follows. The procedure uses a sample of charged tracks from leptonic and hadronic decays of the $Z^{0}$ that satisfy the criteria $p_{1}>0.25 \mathrm{GeV} / c,\left|d_{0}\right|<1 \mathrm{~cm}$, and $\sigma\left(d_{0}\right)<0.1 \mathrm{~cm}$. Here $p_{\mathrm{t}}$ is the track momentum in the plane orthogonal to the beam direction, $d_{0}$ is the distance of closest approach of the track to the coordinate origin, and $\sigma\left(d_{0}\right)$ is the estimated uncertainty in $d_{0}$. Such tracks from consecutive events are accumulated until there are at least 100. A $\chi^{2}$ fit is then performed for a vertex in the $x-y$ plane. If any tracks contribute more than 10 to the $\chi^{2}$ of the vertex fit, the worst track is discarded and a new fit performed. This procedure is iterated until all tracks have $\chi^{2}<10$ with respect to the vertex. Given 30 such sets of vertex coordinates, which correspond typically to about $200 Z^{0}$ decays, a weighted mean is taken to determine the beam position. The typical statistical accuracy of the mean $x(y)$ position is $15(10) \mu \mathrm{m}$. After the set of centroid coordinates for a given run is obtained, adjacent measurements that are statistically compatible are combined. This process results in 1 or 2 beam position measurements per run. The beam coordinates for the various 1990 OPAL runs are plotted in fig. 1.

Once the beam coordinates are known, the distribution of impact parameters of tracks with small $\sigma\left(d_{0}\right)$ can be used to determine the intrinsic size of the beam crossing region. The vertical beam size is expected to be very small and so the width of the $d_{0}$ distribution of horizontal tracks can be taken as a measure of the tracking resolution. This width is subtracted in quadrature from the width measured for vertical

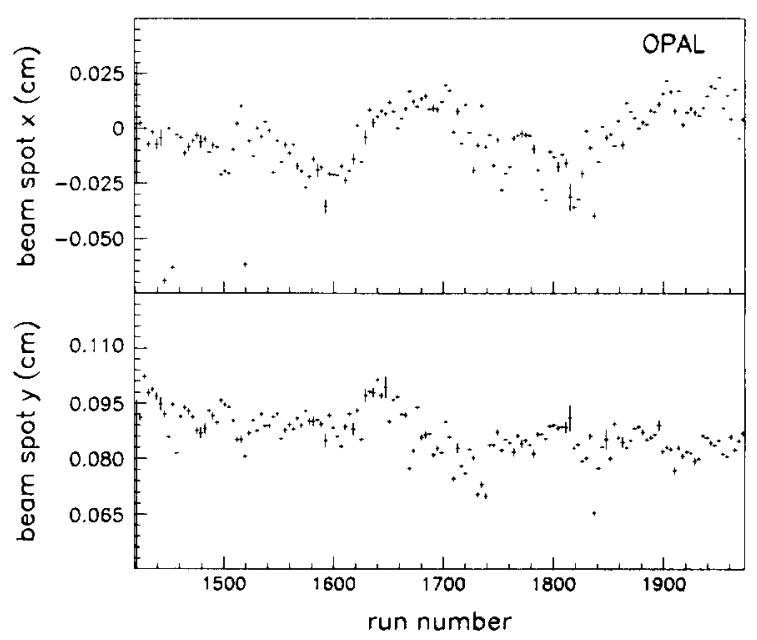

Fig. 1. Distributions of the beam coordinates for the 1990 OPAL data. The error bars indicate the statistical precision on the mean of each determination.

tracks to calculate an average value of the beam spot size in the $x$-direction of $\sigma_{\mathrm{b} x}=(157 \pm 5) \mu \mathrm{m}$. The width in $z$ implies a beam size of $\sigma_{\mathrm{b} z}=(1.12 \pm$ $0.02) \mathrm{cm}$. These determinations are in excellent agreement with the range of values expected from the LEP beam optics (assuming head-on collisions and gaussian bunch shapes) of $\sigma_{\mathrm{b} x}=155-190 \mu \mathrm{m}, \sigma_{\mathrm{b} y}=6-$

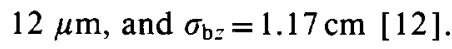

In the following analyses, and in the Monte Carlo simulation of event samples, the beam spot size used is $\sigma_{\mathrm{b} x}=165 \mu \mathrm{m}, \sigma_{\mathrm{b} y}=8 \mu \mathrm{m}, \sigma_{\mathrm{b} z}=1.167 \mathrm{~cm}$ to which are added, in quadrature, the errors on the beam position estimation. The parameters of every track are redefined with respect to the measured average beam position.

\section{Lifetime measurement by the impact parameter method}

The impact parameter, $d_{0}$, of a track coming from a tau decay is related to the tau decay length, $l$, and the angle, $\psi$, between the decay track and the tau direction by $d_{0}=l|\sin \psi|$. The convention is to assign a positive sign to the impact parameter if the decay product track crosses the tau direction in the same hemisphere as the track, and a negative sign otherwise. This convention results in a signed impact parameter distribution shifted positive from zero, and 
allows the lifetime of the tau lepton to be measured by comparing the observed shift to that predicted by the Monte Carlo.

This impact parameter analysis has been applied to the 1-prong tau decays in order to remain statistically independent of the 3-prong decay length analysis presented in the following section. The event thrust axis, calculated using the charged tracks only, approximates the tau direction and is used to sign the $d_{0}$ measurements. Events in which both taus decayed to a single track of the same measured charge have been rejected. After removal of these events, $0.6 \%$, the data sample contains 8245 1-prong tau decays. The 1-prong tau decay tracks to be used in the lifetime measurement are then required to have (i) at least 6 hits out of the 12 axial wires in the vertex chamber, which eliminates 212 tracks; (ii) at least half of the geometrically possible hits in the jet chamber, which eliminates 38 tracks; (iii) the total error on $d_{0}$ less than $0.1 \mathrm{~cm}$, which eliminates 8 very poorly measured tracks. These requirements result in a final sample of 7987 1-prong tau decay tracks. The average error due to the track fit including multiple scattering is $70 \mu \mathrm{m}$, and the average contribution due to the beam spot size is $105 \mu \mathrm{m}$.

In the ideal situation a track coming from the tau decay will always cross the original tau path in front of the interaction point and will always be assigned a positive impact parameter. There are two effects that give rise to negative entries in the signed $d_{0}$ distribution: (i) the approximation of the tau direction by the thrust axis and (ii) impact parameter measurement errors including the assumption that the tau is produced at the centre of the beam ellipse. Impact parameter distributions have been formed using the Monte Carlo generator information, after including the effects of detector acceptance, but before the simulation of detector resolution. The mean of the distribution of impact parameters signed using a thrust axis constructed with the charged particle momenta is significantly lower than the mean of the distribution formed using the true tau direction to sign the impact parameters. The magnitude of this reduction in the mean depends on the topology of the event. For those events in which both taus undergo a 1-prong decay, this reduction is $28 \%$. For the events in which one of the taus decays to 3 charged particles, a larger fraction of the tau momentum is visible, and the reconstructed thrust axis better approximates the actual tau direc- tion. Thus the reduction in the mean of the 1-prong impact parameter distribution in this case is only $10 \%$. As expected, the resolution of the $d_{0}$ measurements serves to broaden the impact parameter distribution but does not significantly shift the mean. After including the full simulation of the detector, the mean of the signed $d_{0}$ distribution is the same, to within $2 \%$, as that calculated before detector resolution. The signing errors introduced by the thrust axis calculation are the most important component in determining the mean of the signed impact parameter distribution.

The tau lifetime is determined by comparing the signed impact parameter distribution from the data with the corresponding distribution from the large Monte Carlo sample described above. A simple mean of the distribution is sensitive to entries with large $\left|d_{0}\right|$ values, arising from mismeasurements not well modeled by the Monte Carlo. Instead, the trimmed mean, $\bar{x}$, is used because it is less sensitive to the tails of the distribution. The trim parameter, $t$, has a range from 0 to 1 and is defined so that $t=0.1$ represents a trim of $10 \%$ of the data, $5 \%$ from each of the high and low sides. The uncertainty in the trimmed mean was derived by Nelson to be [13]

$$
\begin{aligned}
s_{\bar{x}}^{2} & =\frac{1}{N(1-t)}\left[s^{2}+\frac{t}{4}\left(\frac{\left(x_{\max }-x_{\min }\right)^{2}}{1-t}\right.\right. \\
& \left.\left.+\left(x_{\max }-2 \bar{x}+x_{\min }\right)^{2}\right)\right] \\
s^{2} & =\frac{1}{N} \sum_{i=N t / 2}^{N(1-t / 2)}\left(x_{i}-\bar{x}\right)^{2},
\end{aligned}
$$

where $N$ is the total number of impact parameters, $x_{i}$ are the individual impact parameters, $x_{\min }$ is the minimum $d_{0}$ after trimming, $x_{\max }$ is the maximum $d_{0}$ after trimming.

The relative statistical uncertainty of the trimmed mean has been calculated, using data and Monte Carlo samples, for various trims. There is a broad minimum about a trim value of $t=0.1$, which is the trim value chosen. The tau lifetime is determined from the ratio of these trimmed means,

$\tau_{\exp }=\tau_{\mathrm{MC}} \frac{\bar{x}_{\text {exp }}}{\bar{x}_{\mathrm{MC}}}$,

where $\tau_{\mathrm{MC}}=0.303 \mathrm{ps}$ was the value used in the Monte 


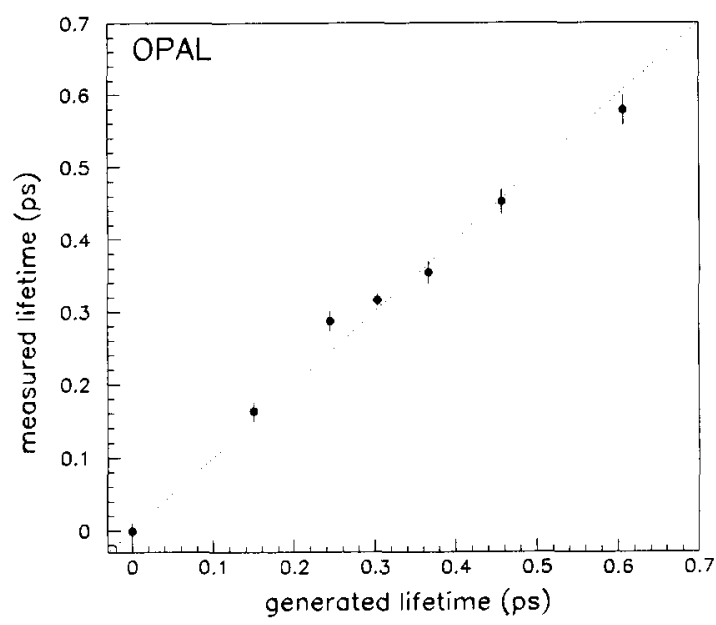

Fig. 2. Measured lifetimes of Monte Carlo samples generated with different lifetimes when compared to the high statistics Monte Carlo generated with the world average lifetime The dotted line is a line of unit slope through the origin. The $\chi^{2}$, calculated with respect to this line, is 14.6.

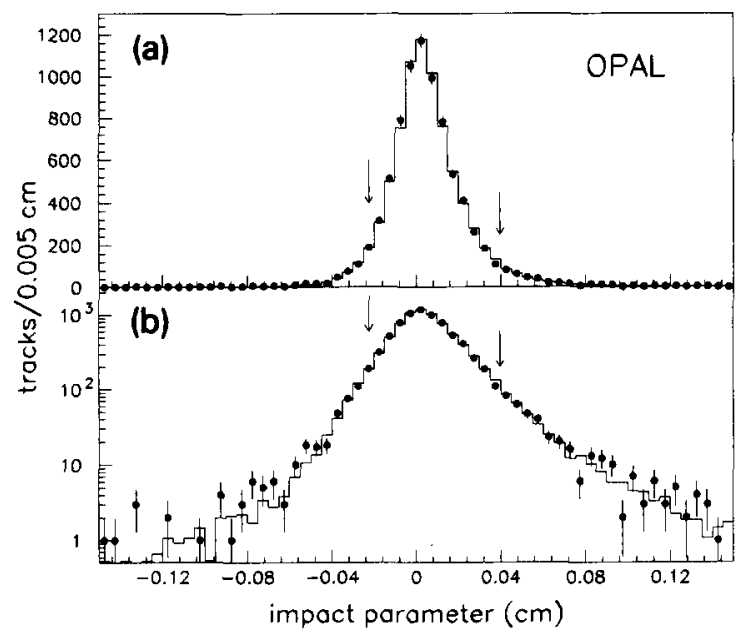

Fig. 3. The 1-prong signed impact parameter distribution for the 1990 OPAL data compared to the Monte Carlo distribution, shown on (a) linear scale, and (b) log scale. The arrows indicate the cutoff values for a $10 \%$ trim.

Carlo generation. As a check of the procedure, the trimmed mean of the signed $d_{0}$ distribution has been determined for each of the several Monte Carlo samples generated with different lifetimes between 0 and $0.6 \mathrm{ps}$. The measured lifetimes are consistent with the generated lifetimes as shown in fig. 2 .

Fig. 3 shows the signed impact parameter distribu- tion for the 1-prong data sample compared to Monte Carlo. The trimmed mean is $45.1 \pm 1.9 \mu \mathrm{m}$ and that for the corresponding Monte Carlo distribution is $47.2 \pm$ $0.7 \mu \mathrm{m}$. The derived, uncorrected tau lifetime is $\tau_{\tau}=$ $0.290 \pm 0.013 \mathrm{ps}$, where the error is statistical only.

The stability of the lifetime measurement has been examined as a function of three kinematic variables: (i) $\left|\cos \theta_{\text {thrust }}\right|$, (ii) azimuthal angle of the track, and (iii) the track momentum. No statistically significant variation is observed.

The signed $d_{0}$ distribution for the data contained a small number of entries in excess of the Monte Carlo prediction far out in the tails. Two detector resolution effects are found to contribute to these tails: (a) tracks with only 6-8 vertex chamber hits, and (b) events with several extra tracks that are eliminated by the original good track definition cuts. Although these effects contribute significantly to the tails of the $d_{0}$ distribution, they do not introduce a bias in the trimmed mean.

The lifetime has also been examined for a possible bias arising from the small difference in the Monte Carlo distribution of event topologies, $1: n$, where $n=1-5$, from that observed in the data. The Monte Carlo trimmed mean has been calculated by recombining the individual trimmed means of each topology according to the topology fractions actually seen in the data. This recalculation is in agreement with the original trimmed mean to within the statistical uncertainty of the Monte Carlo sample, and no correction has been applied.

Because the signed $d_{0}$ distribution is asymmetric, the trimmed mean value varies from the simple arithmetic mean, $t=0$, to the median, $t=1$. Any difference in the signed $d_{0}$ distribution asymmetry between the Monte Carlo and the data could lead to a small bias in the trimmed mean determination and a corresponding systematic error in the lifetime. The effect of such a possible asymmetry difference can be seen by calculating the lifetime over the full range of possible trim values, as shown in fig. 4 . The lifetime is constant to within $\pm 1.7 \%$, for trims larger than $t=0.01$.

The lifetime has been determined using a thrust axis calculated with charged tracks only. The trimmed mean analysis requires a good simulation of the experimental thrust axis properties. To estimate the systematic error due to this simulation, the lifetime has been determined using an alternative axis calculated 


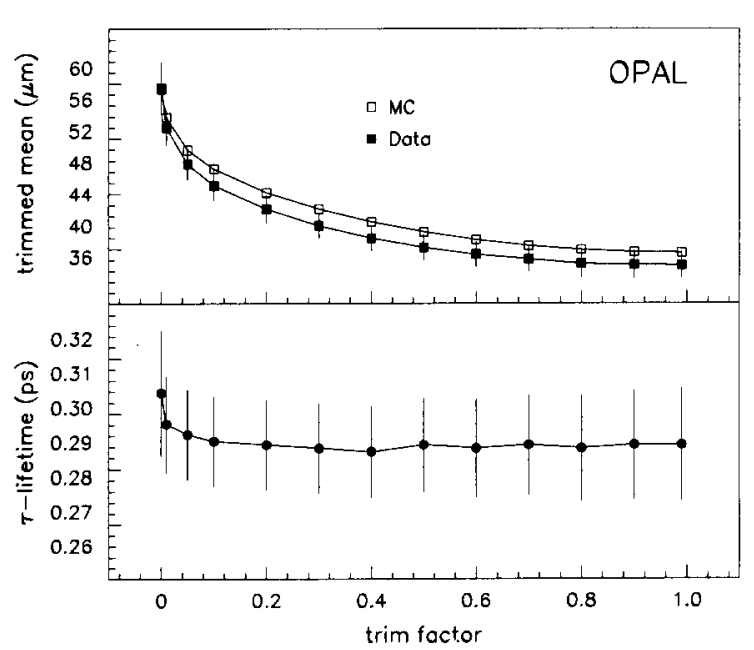

Fig. 4. The trimmed means for both data and Monte Carlo, and the measured lifetime as a function of trim.

by combining the charged track and electromagnetic measurements. In order to ensure an accurate simulation of the cluster information, the clusters included in the calculation are required to have an energy greater than $1 \mathrm{GeV}$ and to be within $0.4 \mathrm{rad}$ of the charged track thrust axis in the $x-y$ plane. If this alternative axis is used for signing the impact parameters, the lifetime changes by $4.1 \%$ which is taken as a systematic uncertainty in the lifetime from the knowledge of the thrust axis.

There is a background contribution of $(2.4 \pm 1.2) \%$ in the 1-prong tau decay sample. Although the sources contributing to this background contain no lifetime information, and thus are expected to have symmetric signed $d_{0}$ distributions about zero, the $10 \%$ trim procedure for the tau sample leads to asymmetric cuts and results in a nonzero trimmed mean of the background component. This effect was measured using a data sample of $4500 \mu^{+} \mu^{-}$pairs and yields $(21.3 \pm$ 1.2) $\mu \mathrm{m}$. After correction for the non- $\tau^{+} \tau^{-}$background in the data, the trimmed mean of the 1-prong tau decay signed $d_{0}$ distribution is $(45.7 \pm 1.9) \mu \mathrm{m}$ which results in a lifetime of $\tau_{\tau}=(0.293 \pm 0.013)$ ps. The magnitude of this correction is $+1.3 \%$ and its systematic uncertainty due to the uncertainty in the amount of background is $\pm 0.7 \%$.

The trimmed means for individual tau decay modes have been determined in the Monte Carlo, and the trimmed mean has been recalculated to reflect the small differences between the branching ratios in the KORALZ generator and the current measured world average values. This results in a change of $0.7 \%$ in the lifetime determination which is taken as a systematic error.

In summary, the following systematic errors are assigned to the impact parameter method:

- variation of trim factor: $\pm 1.7 \%$;

- simulation of thrust axis: $\pm 4.1 \%$;

- uncertainty in tau sample background: $\pm 0.7 \%$;

- uncertainties in decay mode branching ratios:

$\pm 0.7 \%$;

- the total systematic error combining each of these contributions in quadrature is $\pm 4.5 \%$.

The final result for the tau lifetime from the trimmed mean analysis of the signed impact parameter distribution of the 1-prong tau decays is

$\tau_{1}=0.293 \pm 0.013$ (stat.) \pm 0.013 (syst.) ps.

\section{Lifetime measurement by the decay length method}

A tau lifetime measurement has been performed in 3-prong decays using a two-dimensional decay length fit [14]. The vertex fit of the tracks is combined with beam spot information and the tau flight direction, which is assumed to be the thrust axis, to determine the most probable value of the $x-y$ decay length, $l_{x y}$, and its uncertainty, $\sigma_{l}$. For details, see the appendix. The three dimensional decay length, $L_{x y z}$, and error, $\sigma_{L}$, are obtained from the relations $L_{x y z}=l_{x y} / \sin \theta$, and $\sigma_{L}=\sigma_{l} / \sin \theta$, where $\theta$ is the tau polar angle.

The $\tau$ selection previously described results in an initial sample of 14753 -prong $\tau$ decays. To remove contamination of the 3-prong tau decays by photon conversions and by decays of $\mathrm{K}^{0} \rightarrow \pi^{+} \pi^{-}$, any charged track pair with a reconstructed secondary vertex that contributes to the mass regions $m\left(\mathrm{e}^{+} \mathrm{e}^{-}\right)<0.05$ $\mathrm{GeV}$ or $0.473<m\left(\pi^{+} \pi^{-}\right)<0.523 \mathrm{GeV}$ is rejected. All 1341 remaining 3-track candidates have been fit to common vertices in the $x-y$ plane to determine the best values of each tau decay position and its associated error matrix. The decay vertex is estimated with a precision of approximately $0.3 \mathrm{~cm}$ along the flight direction and approximately $50 \mu \mathrm{m}$ transverse to the flight direction. 
The decay length, its error, and the $\chi_{\min }^{2}$ probability are calculated using the formalism described in the Appendix. Since the 1990 LEP data have not been taken at the same beam energy, all calculated lengths have been normalised to a nominal centre of mass energy of $91.160 \mathrm{GeV}$. Cuts on the 3-prong vertex fit probability of $1 \%$ and on the two-dimensional decay length fit probability of $1 \%$ have been applied. This results in 1009 3-prong decays.

The distribution of decay lengths is taken to be the convolution of an exponential smeared by a resolution function which is assumed to be a gaussian whose width varies from event to event according to the uncertainty on the measured decay length. The probability of observing a decay length $L_{i}$ having measurement uncertainty $\sigma_{i}$, under the hypothesis of a parent distribution decay length $L_{0}$, is

$$
\begin{aligned}
& P\left(L_{i}, \sigma_{i}, L_{0}, s\right)=\frac{1}{L_{0} s \sigma_{i} \sqrt{2 \pi}} \\
& \quad \times \int_{0}^{\infty} \exp \left(-x / L_{0}\right) \exp \left(\frac{-\left(x-L_{i}\right)^{2}}{2 s^{2} \sigma_{i}^{2}}\right) \mathrm{d} x \\
& \quad=\frac{1}{2 L_{0}} \exp \left(\frac{s^{2} \sigma_{i}^{2}}{2 L_{0}}-\frac{L_{i}}{L_{0}}\right) \operatorname{erfc}\left[\frac{1}{\sqrt{2}}\left(\frac{s \sigma_{i}}{L_{0}}-\frac{L_{i}}{s \sigma_{i}}\right)\right],
\end{aligned}
$$

where, in addition to the decay length $L_{0}$, a common scale factor $s$ for the errors $\left(\sigma_{i}\right)$ is included as a fit variable. This provides an allowance for the possibility that the fit errors have been incorrectly estimated and assumes explicitly that a uniform scale factor is adequate. The product of these probabilities for all entries included in the fit is maximised to produce the best estimate of the decay length.

The maximum likelihood fit is quite sensitive to excesses of events in the tails of the decay length distribution. Quality cuts for selecting the measurements included in the fit have been carefully chosen to be unbiased with respect to decay length. In addition, a cut window in the decay length has been used to eliminate the effects of a few poorly-reconstructed events remaining in the tails after the quality cuts have been applied. In this case, eq. (1) must be modified by the inclusion of a normalisation factor to take account of the reduced range of the decay lengths in the probability integral [15]. This factor becomes important whenever the window causes a significant fraction
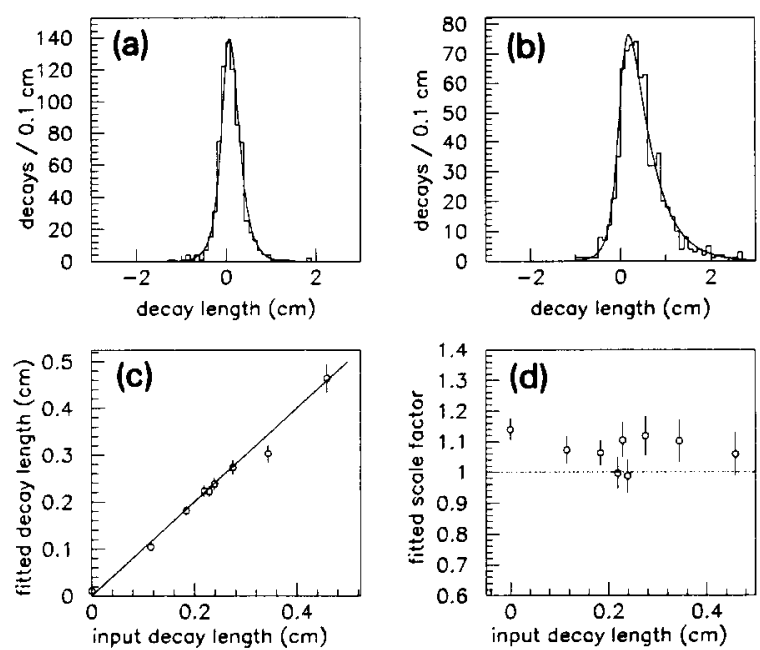

Fig. 5. Maximum likelihood fits to Monte Carlo 3-prong decay length distributions generated with different tau lifetimes. (a) and (b) show the fits for input lifetimes 0.5 and 2.0 times the current world average lifetime of $0.303 \mathrm{ps}$. (c) shows the fitted decay length versus input decay length. The line has unit slope and passes through the origin. (d) shows the fitted scale factors versus the input length. It indicates that the errors in the Monte Carlo are slightly underestimated.

of the entries to be removed. For the lifetime determination a decay length window of $\left[L_{\min }, L_{\max }\right]=$ $[-0.6,+1.8] \mathrm{cm}$ is chosen, based on a Monte Carlo study that compared the statistical significance of different window choices. In addition, a cut on the maximum allowed magnitude of the decay length error was studied for its effect on the fitted value of the decay length. The determination was found to be insensitive to this cut since large error events carry little weight, and a value of $0.6 \mathrm{~cm}$ has been used.

As a check on the procedure, the decay length analysis has been performed on Monte Carlo data sets generated with different lifetimes. Two of these fits, for samples with 0.5 and 2.0 times the current world average tau lifetime of $0.303 \mathrm{ps}$ are shown in figs. 5a, $5 \mathrm{~b}$. The results of fits to all samples are summarised in figs. $5 \mathrm{c}, 5 \mathrm{~d}$. The calibration curve of measured decay length versus input decay length shows no bias.

A maximum likelihood fit to the 838 decays with decay length error less than $0.6 \mathrm{~cm}$ within the window cut $[-0.6,+1.8] \mathrm{cm}$ yields decay length and scale factor

$L_{0}=0.247 \pm 0.013 \mathrm{~cm}, \quad s=1.277 \pm 0.062$. 


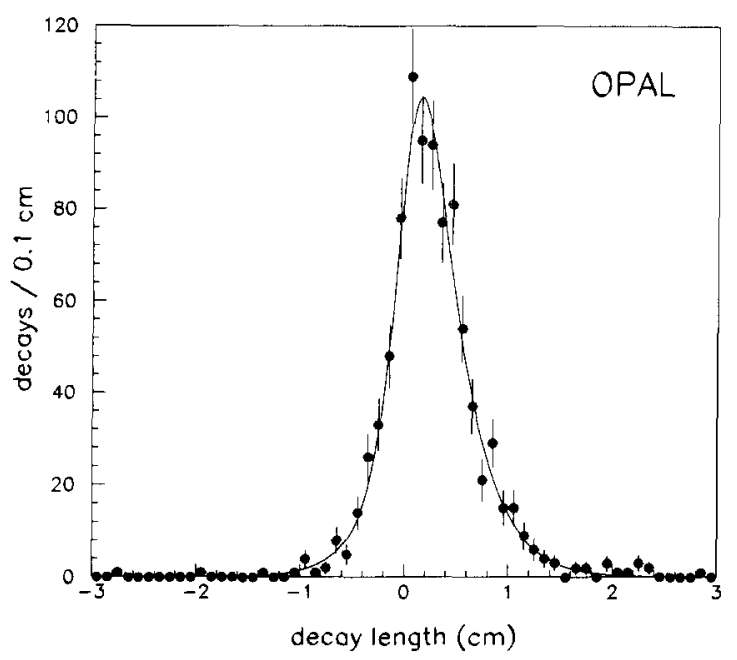

Fig. 6. The maximum likelihood fit to the 3-prong decay lengths with length error less than $0.6 \mathrm{~cm}$. The fit was for the 838 events which had decay lengths within the range $[-0.6,+1.8] \mathrm{cm}$. Approximately $3.5 \%$ of the data lies outside of the fitted range.

The result is shown in fig. 6 . A fit quality is given by the $\chi^{2}$ of the overlaid plotting function with respect to the histogram values, which is 12.8 for 21 degrees of freedom. The average Lorentz boost factor, corrected for radiation, needed to convert a measured decay length to a particle lifetime has been calculated with the Monte Carlo to be $\langle\beta \gamma\rangle=25.32 \pm$ 0.01 . The fitted value of the decay length, taken together with this boost factor, gives a measured lifetime of $0.325 \mathrm{ps}$. As a check on the result, the trimmed mean and the trimmed weighted mean of the threedimensional decay length distribution have been calculated and the lifetime inferred from a calibration curve of the Monte Carlo data sets. If the errors on the parameters of the straight line fits are ignored, the data values of trimmed mean and trimmed weighted mean correspond to decay lengths of $0.256 \pm 0.016 \mathrm{~cm}$ and $0.240 \pm 0.015 \mathrm{~cm}$, respectively. These values are in good agreement with the result from the maximum likelihood fit.

Many of the selection parameters have been varied and further restrictions made on data quality to look for any possible systematic bias on the lifetime. In particular, the variation with $\cos \theta_{\text {thrust }}, \phi_{\text {thrust, }}$, the $\chi_{\min }^{2}$ probability of the decay length fit, the tau decay vertex fit probability, the event topology, and the run number have been studied. In addition, the sample has been further restricted to those decays that include measurements in the vertex and $z$-chambers. No statistically significant variation in the lifetime is observed. The following factors which can contribute to a systematic uncertainty have been studied. Instead of the charged track thrust axis, a thrust axis has been calculated from both the charged tracks and electromagnetic clusters. This gives some indication of the sensitivity of the result to the thrust axis calculation, and leads to a systematic uncertainty of $\pm 2.3 \%$. The beam position is subject to possible offsets and the beam size to possible underestimation. Reasonable variations in both these values have been investigated, and a systematic uncertainty of $\pm 1.2 \%$ assigned. The photon conversion mass cut of $50 \mathrm{MeV}$ on the $\mathrm{e}^{+} \mathrm{e}^{-}$track pair candidate has been varied, including a null cut, and a systematic uncertainty of $\pm 1.6 \%$ determined. The multihadron contamination to the original 3-prong tau decay sample has been estimated to be $(1.2 \pm 2.3) \%$. The effective contamination of those decays selected for the maximum likelihood fit of the decay length distribution is found to be $(0.6 \pm 1.2) \%$, and this component has a mean decay length of $-0.005 \mathrm{~cm}$, consistent with zero. Thus, the measured result was adjusted by $+0.6 \%$ and a systematic error of $\pm 1.2 \%$ was assigned.

In summary, the following systematic errors are assigned to the decay length method:

- choice of thrust axis: $\pm 2.3 \%$;

- uncertainty of beam position/size: $\pm 1.2 \%$;

- photon rejection cut: $\pm 1.6 \%$;

- multihadron background: $\pm 1.2 \%$;

- the total systematic error combining each of these contributions in quadrature is $\pm 3.3 \%$.

The final result for the tau lifetime from this decay length analysis of the 3-prong tau decay is

$\tau_{3}=0.327 \pm 0.017$ (stat.) \pm 0.011 (syst.) ps.

\section{Summary}

After combining the statistical and systematic errors for each analysis in quadrature, the weighted average lifetime is calculated to be

$\tau_{\tau}=0.308 \pm 0.013$ ps. 
Such a procedure assumes that the systematic errors associated with the thrust axes in the two methods are not correlated. The observed shifts in the two lifetime analyses are in opposite directions which suggests that the errors are anti-correlated and hence the error estimate is conservative.

The OPAL measurement of the $\tau$ lifetime is consistent with the present world average of $0.303 \pm 0.008 \mathrm{ps}$ and other recent measurements [16].

\section{Appendix. Decay length formalism}

Summarised below is the two-dimensional formalism to determine the decay length of short lived particles [14]. There are three essential inputs:

(i) the average beam position $\left(x_{\mathrm{b}}, y_{\mathrm{b}}\right)$ in the transverse plane and the spatial extent of the beam $\left(\sigma_{\mathrm{b} x}\right.$, $\left.\sigma_{\mathrm{b} v}\right)$,

(ii) the measured decay vertex of the tau $\left(x_{\tau}, y_{\tau}\right)$ and its associated errors $\left(\sigma_{\tau x}, \operatorname{cov}\left[\sigma_{\tau x} \sigma_{\tau y}\right], \sigma_{\tau y}\right)$,

(iii) an estimate of the tau flight direction. This is represented by the event thrust axis calculated using charged tracks only $\left(t_{x}, t_{y}\right)$, where $t_{x}^{2}+t_{y}^{2}=1$.

The decay length method determines, via a least squares minimisation procedure, the most probable tau decay length $\left(l_{x y}\right)$, the most probable decay position $\left(x_{0}, y_{0}\right)$, and the most probable production point $\left(x_{\mathrm{p}}\right.$,

$\left.y_{\mathrm{p}}\right)$ subject to the directional constraint

$x_{0}=x_{\mathrm{p}}+l_{x y} t_{x}, \quad y_{0}=y_{\mathrm{p}}+l_{x y} t_{y}$.

The solution of the least squares minimisation is as follows. Defining

$V_{\mathrm{b}}=\left(\begin{array}{cc}V_{\mathrm{b} x x x} & 0 \\ 0 & V_{\mathrm{b} y y}\end{array}\right), \quad V_{\tau}=\left(\begin{array}{ll}V_{\tau, x x} & V_{\tau x y} \\ V_{\tau x y} & V_{\tau, y y}\end{array}\right)$,

where the variance matrix elements are, $V_{\mathrm{b} x x}=\sigma_{\mathrm{b} x}^{2}$, etc., and using the following identities:

$x \equiv x_{\tau}-x_{\mathrm{b}}, \quad y \equiv y_{\tau}-y_{\mathrm{b}}, \quad V \equiv V_{\tau}+V_{\mathrm{b}}$,

$D \equiv t_{y}^{2} V_{x x}+t_{x}^{2} V_{y y}-2 t_{x} t_{y} V_{x y}$

then

$l_{x y}=\frac{x t_{x} V_{y y}+y t_{y} V_{x x}-\left(x t_{y}+y t_{x}\right) V_{x y}}{D}$,

$$
\begin{aligned}
& \sigma_{l x y}=\sqrt{\frac{V_{x x} V_{y y}-V_{x y}^{2}}{D}}, \\
& \chi_{\min }^{2}=\frac{\left(x t_{y}-y t_{x}\right)^{2}}{D} .
\end{aligned}
$$

\section{Acknowledgement}

It is a pleasure to thank the SL Division for the efficient operation of the LEP accelerator, the precise information on the absolute energy, and their continuing close cooperation with our experimental group. In addition to the support staff at our own institutions we are pleased to acknowledge the following: Department of Energy, USA; National Science Foundation, USA; Science and Engineering Research Council, UK; Natural Sciences and Engineering Research Council, Canada;

Israeli Ministry of Science; Minerva Gesellschaft; Japanese Ministry of Education, Science and Culture (the Monbusho) and a grant under the Monbusho International Science Research Program; American Israeli Bi-national Science Foundation; Direction des Sciences de la Matière du Commissariat à l'Energie Atomique, France;

Bundesministerium für Forschung und Technologie, FRG;

A.P. Sloan Foundation;

and Junta Nacional de Investigação Científica e Tecnológica, Portugal.

\section{References}

[1] See, e.g., B. Barish and R. Stroynowski, Phys. Rep. 157 (1988) 1 , and references therein.

[2] Particle Date Group, J.J. Hernández et al., Review of particle properties, Phys. Lett. B 239 (1990) 1.

[3] OPAL Collab., K. Ahmet et al., Nucl. Instrum. Methods A 305 (1991) 275.

[4] P. Billoir, Nucl. Instrum. Methods 225 (1984) 352.

[5] OPAL Collab., G. Alexander et al., Measurement of the $Z^{0}$ lineshape parameters and the electroweak couplings of charged leptons, preprint CERN-PPE/9167 , submitted to Z. Phys. C. 
[6] T. Sjöstrand, Comput. Phys. Commun. 39 (1986) 347; JETSET, Version 7.2.

[7] G. Marchesini and B.R. Webber, Nucl. Phys. B 310 (1988) 461; HERWIG, Version 4.3.

[8] M. Böhm, A. Denner and W. Hollik, Nucl. Phys. B 304 (1988) 687;

F.A. Berends, R. Kleiss and W. Hollik, Nucl. Phys. B 304 (1988) 712.

[9] S. Jadach, B.F.L. Ward and Z. Was, Comput. Phys. Commun. 66 (1991) 276.

[10] R. Bhattacharya, J. Smith and G. Grammer, Phys. Rev. D 15 (1977) 3267;

J. Smith, J.A.M. Vermaseren and G. Grammer, Phys. Rev. D 15 (1977) 3280.

[11] J. Allison et al., Comput. Phys. Commun. 47 (1987) 55 ;

R. Brun et al., GEANT 3, CERN report DD/EE/84-1, (1989);

D.R. Ward, The OPAL Monte Carlo program GOPAL, preprint Cavendish-HEP 91/06, Invited talk at MC91 Workshop (Amsterdam, April 1991).
[12] A. Hofmann, private communication.

[13] H.N. Nelson, Ph.D. Thesis, SLAC-Report-322 (October 1987); Note that there is a typographical error in the formula for the trimmed mean error.

[14] MARK-II Collab., J.A. Jaros et al., Phys. Rev. Lett. 51 (1983) 955;

Further details are given in R. Ong and K. Riles, The decay length method, MARK II/SLC note 166 (December, 1986), unpublished.

[15] A.

G. Frodesen, O. Skjeggestad and H. Tofte, Probability and statistics in particle physics, Universitetsforlaget, Bergen, Norway, 1979);

C. Kleinwort, Bestimmung der lebensdauer des $\tau$-leptons mit dem JADE-detektor, Ph.D. thesis (Universität Hamburg, 1988).

[16] L3 Collab., B. Adeva et al., Phys. Lett. B 265 (1991) 451;

DELPHI Collab., P. Abreu et al., Phys. Lett. B 267 (1991) 422. 